Annales Geophysicae (2004) 22: 1103-1113

SRef-ID: $1432-0576 / \mathrm{ag} / 2004-22-1103$

(C) European Geosciences Union 2004

\title{
Diurnal auroral occurrence statistics obtained via machine vision
}

\author{
M. T. Syrjäsuo and E. F. Donovan \\ Institute for Space Research, University of Calgary, Alberta, Canada \\ Received: 21 March 2003 - Revised: 8 August 2003 - Accepted: 1 September 2003 - Published: 2 April 2004
}

\begin{abstract}
Modern ground-based digital auroral All-Sky Imager (ASI) networks capture millions of images annually. Machine vision techniques are widely utilised in the retrieval of images from large data bases. Clearly, they can play an important scientific role in dealing with data from auroral ASI networks, facilitating both efficient searches and statistical studies. Furthermore, the development of automated techniques for identifying specific types of aurora opens up the potential of ASI control software that would change instrument operation in response to evolving geophysical conditions. In this paper, we describe machine vision techniques that we have developed for use on large auroral image data sets. We present the results of application of these techniques to a 350000 image subset of the CANOPUS Gillam ASI in the years 1993-1998. In particular, we obtain occurrence statistics for auroral arcs, patches, and Omega-bands. These results agree with those of previous manual auroral surveys.
\end{abstract}

Key words. Ionosphere (Instruments and techniques) General (new fields)

\section{Introduction}

The spatial structure and temporal evolution of the auroral luminosity distribution result from the time history of the solar wind-magnetosphere interaction and the physics of the magnetosphere-ionosphere interaction. Surveys of optical data obtained from the ground and from space have established the existence of the ever-present auroral oval, as well as variations in its overall brightness distribution and the latitudes of its poleward and equatorward boundaries (Feldstein, 1963; Akasofu, 1966). These are both local time (i.e. "LT") and temporal (i.e. "UT") variations. Simple examples of this variation are that the oval is on average at higher geomagnetic latitudes at dusk and dawn than at midnight (an LT variation), and that the overall distribution moves equa-

Correspondence to: M. T. Syrjäsuo

(mikko@phys.ucalgary.ca) torward and poleward as a consequence of magnetospheric energy loading and dissipation (a UT variation).

Within the oval, the spatial structure and overall brightness also evolve in time. Early auroral research led to the identification of several types of aurora, some that have turned out to be characteristic of the ionospheric footpoint of specific magnetospheric regimes (cf. Feldstein and Elphinstone, 1992), and others indicating ongoing dynamic activity. For example, near the equatorward edge of the evening sector auroral oval there is often proton precipitation in the absence of electron precipitation. This indicates field lines threading the inner magnetosphere, earthward of Central Plasma Sheet (CPS) electron drift paths, and tailward of the inner edge of strong pitch angle scattering of CPS protons (Ejiri, 1978; Sergeev et al., 1983; Samson et al., 1992).

Specific electron auroral morphologies include arcs, patches, and Omega-bands. In this study, we use data from the CANOPUS (Canadian Auroral Network for the OPEN Program Unified Study) Gillam All-Sky Imager (ASI) (Rostoker et al., 1995; Donovan et al., 2003). The instrument's characteristics and typical data are discussed below. At this point, we define the auroral features mentioned here in terms of their appearance in a single ASI image. We define arcs to be elongated east-west aligned auroral structures that stretch across most or all of the ASI field-of-view (FOV), with thicknesses (at $\sim 110 \mathrm{~km}$ ) ranging from several to tens of kilometres. This definition is consistent with that used in previous manual statistical studies of auroral arc orientation, occurrence, and thickness (e.g. Stringer and Belon, 1967; Gustafsson, 1967; Akasofu et al., 1972; Nevanlinna and Pulkkinen, 2001; Knudsen et al., 2001). Omega-bands are distortions in the poleward boundary of the diffuse electron aurora, with azimuthal scale sizes that are typically comparable to an ASI FOV (Akasofu and Kimball, 1964; Opgenoorth et al., 1983). Patchy auroras consist of amorphous emission structures with temporally evolving boundaries and scale sizes on the order of tens to hundreds of kilometres. During patchy auroras, the ASI FOV typically contains numerous patches. 
Over the last forty years, a picture of the global morphology has emerged on the basis of extensive ground and space-based optical observations. This consists of the auroral oval, centred roughly on the geomagnetic pole, with a typical latitudinal extent of 5-10 and an equatorward boundary that is typically at $65^{\circ}$ magnetic latitude at midnight and at somewhat higher latitudes at other local times (Feldstein, 1963; Akasofu, 1966). Arcs are a pervasive morphology, occurring at essentially all local times (e.g. Stringer and Belon (1967); Gustafsson (1967)), from the poleward boundary of the oval equatorward to the proton and/or electron isotropic boundaries (Lyons et al., 1988; Yahnin et al., 1997). Statistical studies indicate that arc occurrence peaks on the night side (i.e. Nevanlinna and Pulkkinen (2001)). Omega-bands are morning sector phenomena that occur either following or during substorm activity in the midnight sector (Akasofu et al., 1972; Opgenoorth et al., 1983, 1994). Patchy auroras occur in the late morning to dawn sector and under a range of geomagnetic conditions.

We restrict our definition of an auroral arc to elongated forms which are more or less L-shell aligned (i.e. east-west). The conditions under which arcs form typically last tens of minutes to several hours. This can be at least inferred from plots showing the location and alignment of arcs in the survey papers of Stringer and Belon (1967) and Gustafsson (1967). In recent years, north-south aligned auroral features have received considerable attention due to their demonstrated association with transient magnetospheric phenomena (Henderson et al., 1998; Zesta et al., 2000). While many north-south structures share the same fundamental morphology (i.e., they are elongated discrete auroral features) as typical east-west aligned auroral arcs, they are considerably more rare and are in general transient, lasting only one to several minutes.

The schematic diagram in Fig. 1 illustrates the essence of the Akasofu auroral distribution. This picture was arrived at on the basis of early ground-based auroral surveys and contains phenomena that are ever-present (i.e. the oval), frequently occurring and lasting for periods of hours or more (i.e. arcs and patches), and more infrequent and typically associated with significant transient magnetospheric events (i.e. Omega-bands and the north-south auroral forms discussed below). On a typical night, a single station (or set of stations) rotates under this distribution. The FOV of the CANOPUS ASI located at Gillam Canada is indicated in Fig. 2, as are the scan planes of the CANOPUS Gillam and Rankin Inlet Meridian Scanning Photometers (MSPs). In Fig. 3, we present keograms constructed using data from the Gillam and Rankin Inlet MSPs, obtained on three different nights. We also include six images from the Gillam ASI. The keograms illustrate the typical diurnal variation associated with motion under the auroral oval, most notably in the auroral "motion" from higher to lower to higher latitude as the stations sweep through from dusk to midnight to dawn. Also clearly visible in the keograms are substorm associated changes in the auroral latitude and brightness distributions. Changes over each night in the character of the discrete auroral features reflect a combination of diurnal variation and geomagnetic activity. The six images are examples of arcs $(A \& C)$, patches (B \& D), a north-south auroral structure (E - see below), and an Omega-band (F).

In recent years, the digital ASI has become an important tool in auroral and magnetospheric physics. As increasingly large ASI arrays, such as MIRACLE (Syrjäsuo et al., 1998) and NORSTAR (Donovan et al., 2003) operate with constantly increasing frame rates and CCD size, the size of the resulting data set is becoming formidable. At the present time, the MIRACLE and NORSTAR arrays deliver upwards of 6000000 and 3000000 images annually. Despite the advances in computer science, ASI images are mostly analysed manually. This approach is well motivated especially if the data are used, for example, in supporting satellite measurements, but does not fully utilise the whole data set. In general, analysing a large number of auroral images manually for a statistical study can be done (e.g. Nevanlinna and Pulkkinen, 2001; Knudsen et al., 2001), but repeated analysis to refine results is difficult to justify. Furthermore, newer data sets are large enough that manual studies are not practical.

In this paper, we describe automated pattern recognition (herein referenced to as "machine vision") techniques that we are developing for application to auroral studies. Furthermore, we present the results of applying these techniques to the CANOPUS ASI data set, delivering in particular the local time occurrence statistics of auroral arcs, patches and Omega-bands.

\section{Machine vision approach to image classification}

There is clearly strong motivation for developing techniques for automatic classification of ASI images. Such tools could be applied to large data sets in statistical studies of the aurora, to cull enormous data sets quickly with the objective of identifying auroral events of a particular type, and to quantify the temporal evolution of the auroral distribution. Developing such algorithms would also further clarify our understanding of the aurora by forcing us to further develop what are presently somewhat subjective definitions of auroral type (for example, arc, patch, Omega-band, and north-south structure), making them more objective and suitable for quantitative studies.

In this paper, we describe our initial attempts to develop and systematically apply automated pattern recognition techniques to a 350000 image subset of the CANOPUS Gillam ASI data obtained between 1993 and 1998. More specifically, we developed a "training set" by manually identifying good examples of each of the three auroral forms of interest here (i.e. arc, Omega-band, and patchy). We use techniques from the fields of pattern recognition and content-based image retrieval in order to classify each image as belonging to one of the three classes (arcs, patches, and Omega-bands), or as "other". Note that we attempt to separate east-west arcs from north-south structures by incorporating a quantitative assessment of orientation into our analysis. Our approach is to identify "features" that can be quantified (a simple 


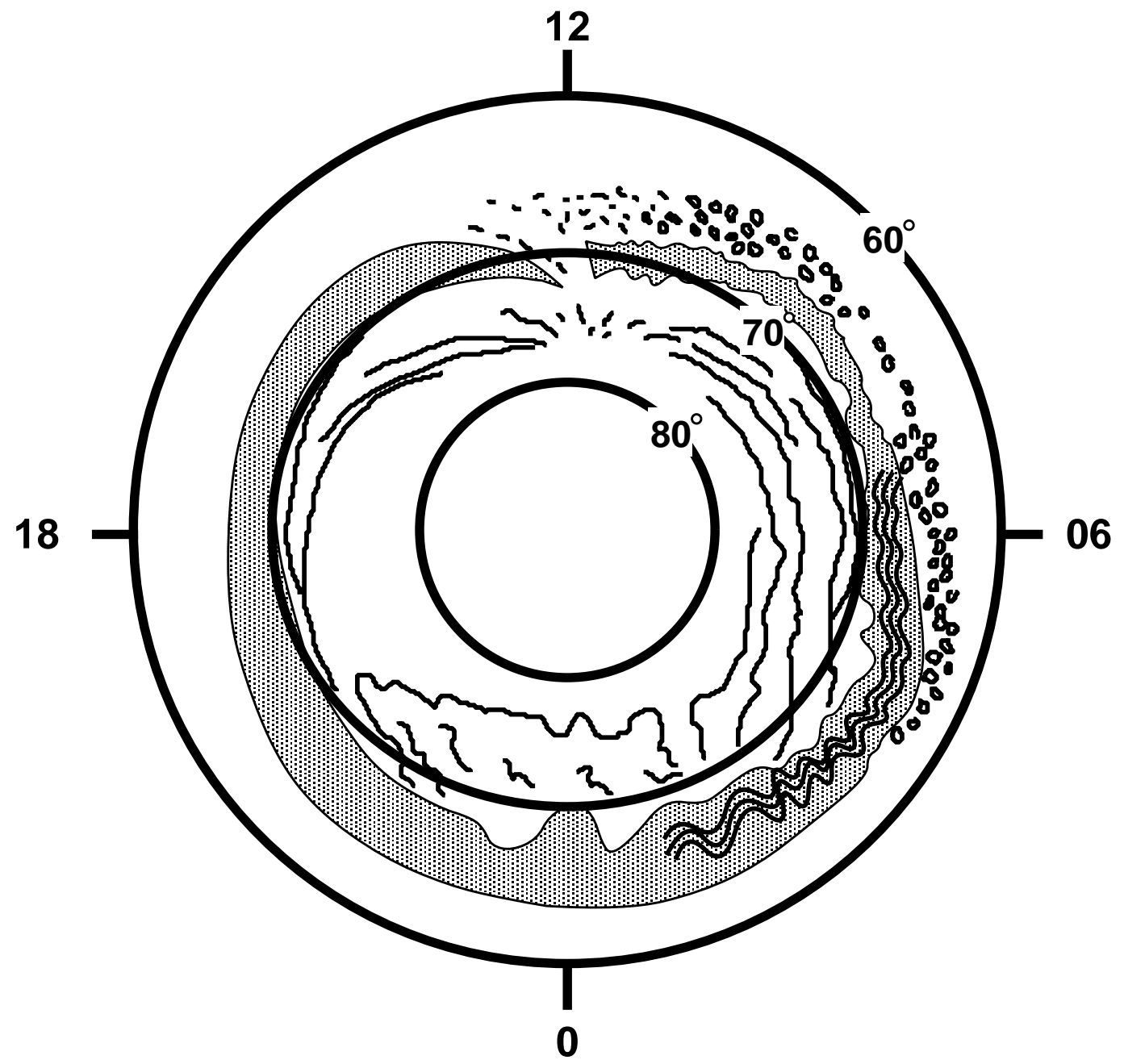

Fig. 1. Schematic of auroral distribution modified slightly from Akasofu (1966). Note that the original figure was constructed on the basis of ground-based observations made before global imaging from space was possible. As such, it incorporates a mixture of diurnal (local time) and temporal (universal time) variations.

example of a feature is average brightness), and that correspond to basis vectors that span a hyperspace that we call our "feature space". In this way, a vector representation is generated for each image in the overall data set. The objective is to develop features such that image-image distance in feature space is a meaningful way of determining how similar the images are to each other in terms of our general understanding of auroral morphology.

In principle, in a well constructed feature space, different hypervolumes correspond to different types of aurora. Having a training set consisting of several hundred auroral images that have been classified as arcs, patches, or Omegabands makes it possible to identify these hypervolumes. For any new image - referred to as a "query image" - its location in the feature space can be determined. Then, the image is classified based on the training set images that are closest to it in the feature space. If most or all of the closest training images are, for example, arcs, then the query image is classified as an arc. The same is true for patches and Omega-bands. The effectiveness of such image classification is strongly affected by the quality of the training set, and the features chosen upon which feature space is constructed. Furthermore, as in the case of polynomial fitting of data, it is more preferable to utilise lower, rather than higher dimensional representations.

In an earlier study, we utilised a simple version of our present approach to separate images that contained, and did not contain aurora (Syrjäsuo and Donovan, 2002). We found that a two-dimensional feature space (average brightness and peak brightness) had two subvolumes separated by an almost straight line, and that virtually all images on one side of the line contained aurora and all images on the other side were either of blanketing clouds or clear skies absent of aurora.

In what follows, we expand on our earlier work by employing an extended training set, more features, and attempting to classify auroral images as belonging to one of three 
types: arcs, patches, or Omega-bands. In the present study, we restrict our attention to elucidating the diurnal occurrence statistics of these three auroral types.

\section{Instrumentation, data and training set}

The CANOPUS ASI was operated at Gillam Canada (56.4 north and $265.4^{\circ}$ east geodetic; $\sim 67.3^{\circ}$ Invariant) from 1986 through to 2001 (Rostoker et al., 1995; Donovan et al., 2003). This was a typical instrument used in auroral research: a fish-eye lens provided a field-of-view covering the whole sky and, after optical filtering (through $\sim 2 \mathrm{~nm}$ band-pass filters), the image was intensified and captured by a CCD (chargecoupled device) camera. Prior to 1993, the ASI operated in a low spatial resolution mode, delivering images in three scientific wavelength $(558,428$, and $630 \mathrm{~nm})$ at a cadence of three images per minute per filter. During and after 1993, the spatial resolution was increased, the $428 \mathrm{~nm}$ measurements were dropped, and the frame rate decreased to one image per minute per filter. The higher spacial resolution images are $200 \times 200$ pixels with a 16-bit intensity reduced to a quasilogarithmic 8-bit value. The exposure time for $558 \mathrm{~nm}$ images was $1.6 \mathrm{~s}$. In this study, we used more than 350000 images $(558 \mathrm{~nm})$ collected from 1993 to 1998.

We identified four main categories of auroral appearance in the all-sky images:

0. No aurora: no visible auroral activity;

1. Arcs: one or more auroral arcs;

2. Patchy aurora: irregular patches of auroral intensity visible in the whole field-of-view;

3. Omega-band: brighter shapes that resemble those seen when an Omega-band is visible in the field-of-view;

4. Other/Reject: shapes that cannot be put into any of the previous categories, such as diffuse aurora, combinations of different types of aurora, north-south structures, etc.

All images in the category "no aurora" should be rejected before the shape analysis. Fortunately, this can be performed automatically as we demonstrated in Syrjäsuo and Donovan (2002). The method is based on a sample set of images that have been manually classified as containing aurora or not containing aurora, and a two-dimensional feature space as described briefly in the Introduction above. Given an unseen image, we compare the average and maximum brightness to those in the sample set in order to identify most similar images, whose classification can be used to determine the most likely class of the unseen image.

The rejection of "no aurora" category reduces the number of images to be analysed from 350000 to 220000 . From the remaining categories, arcs are the most subjectively distinct and have been located in a less sophisticated automated fashion in an earlier study (Syrjäsuo et al., 2001; Kauristie et al.,
2001). Nevertheless, all categories represent a high-level human concept that must be transferred into a numeric form. In order to capture this human concept, one of the authors (EFD) provided a training set consisting of 258 good quality sample images for the categories of arcs (130), patchy auroras (109) and Omega-bands (19), which we judged to be the most unambiguous auroral types that were frequently encountered in the data set. Sample images from these categories are shown in Fig. 4.

\section{Image content classification}

\subsection{Content analysis and image feature space}

A straight-forward and intuitive approach to image content analysis is to determine the most similar images, for a query image, in the training set. These previously unseen images can then be classified to the class of most similar images in the training set. Humans are experts at detecting similarity, but extracting the similarity automatically is not trivial.

In pattern recognition, a number of representative numeric features are used in classification of data (Therrien, 1989). For example, the average brightness in the image or the histogram of intensity values can be used as features. Usually a single feature does not provide satisfying classification performance, and several features are used instead. The features' form a basis of the multi-dimensional feature space. Each image - or its contents - can be associated with a point in this feature space. For example, using the features average brightness $B_{\text {mean }}$ and maximum brightness $B_{\max }$, the resulting feature space is two dimensional. An image with $B_{\text {mean }}=30$ and $B_{\max }=100$ can be represented by $f=$ $[30,100]$. At the other extreme, a feature vector consisting of all pixel values in the image forms a 40000 -dimensional space $(200 \times 200$ pixels $)$.

Comparing the contents of images is performed by comparing the feature vectors. A mathematically convenient solution is to use distances. In our two-dimensional feature space example above, a distance measure between two images represented by $\boldsymbol{f}^{(1)}=[30,100]$ and $\boldsymbol{f}^{(2)}=[50,250]$ could be the Euclidian distance $S=\left\|f^{(1)}-f^{(2)}\right\|_{2}$. A third image $f^{(3)}=[10,40]$ is closer to $f^{(1)}$ than $f^{(2)}$ and hence, more similar, too. Depending on the features, defining a different distance may be more appropriate.

Intuitively, if two images have similar contents and if the features capture this property, the image locations in the feature space should be close to each other. Conversely, distant points in the feature space should correspond to dissimilar images. Unfortunately, this intuition can break down, because the features do not necessarily capture the human higher-level concept properly. This is illustrated in Fig. 5: using qualitative features representing brightness and "arcness" we have a query image and manually classified arc and non-arc images, which form the training set. We then classify the query image to the class represented by the nearest image in the training set. In the left panel, the query image is 


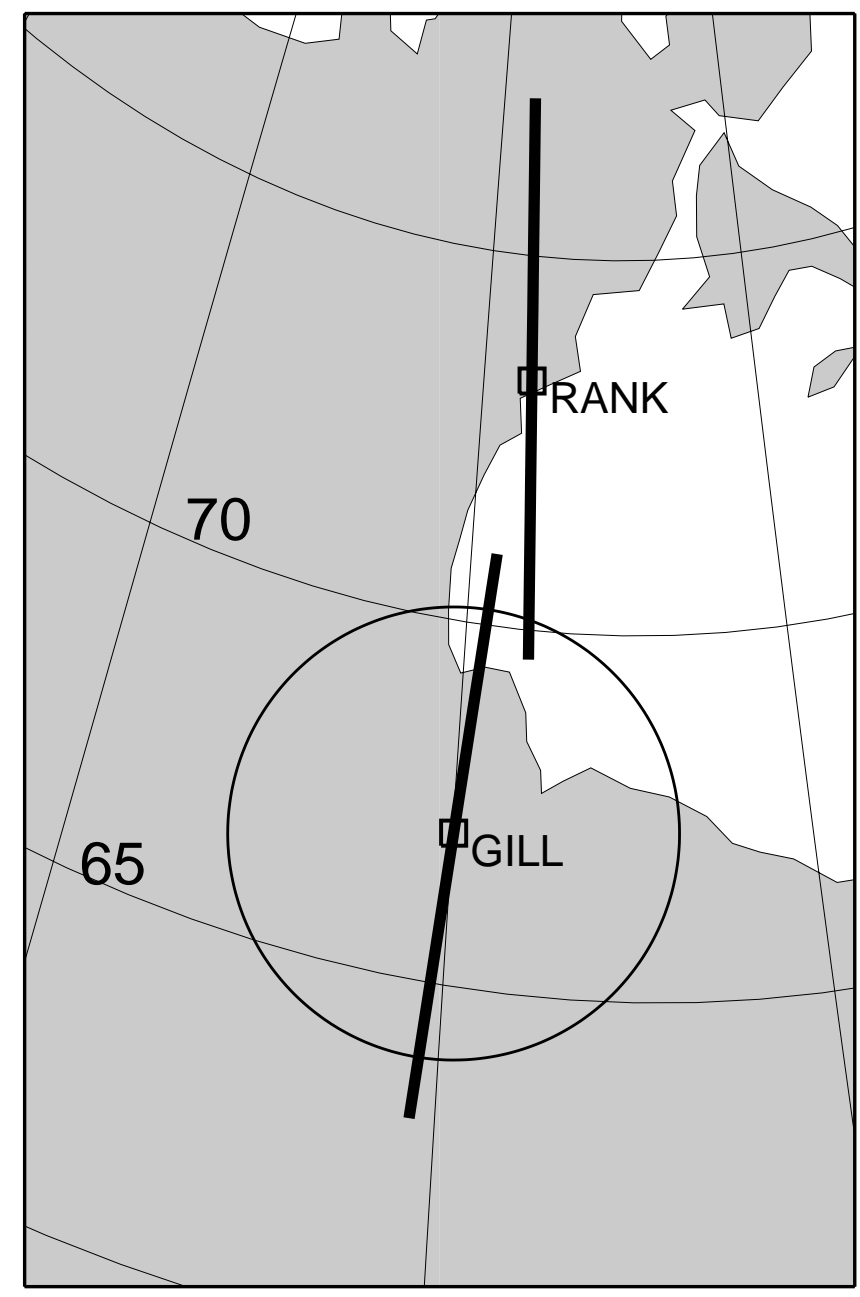

Fig. 2. The ASI used in this study is located at Gillam, Manitoba $\left(56.4^{\circ}\right.$ north, $265.4^{\circ}$ east $)$. The circle indicates the Gillam ASI FOV assuming an emission altitude of $110 \mathrm{~km}$. As well, we also show the FOVs of the Gillam and Rankin Inlet MSPs, again assuming an emission height of $110 \mathrm{~km}$. Contours of constant geomagnetic latitude (at $5^{\circ}$ spacing) and longitude (at $15^{\circ}$ or $1 \mathrm{~h}$ magnetic local time spacing) are also shown.

classified as a non-arc despite its arcness - we are comparing a dim arc to a bright arc. In the right panel, we have sample arcs at all brightness levels and the query image is classified correctly. We included as many images of arcs, patchy auroras and Omega-bands as possible in our training set without excessively tedious manual labour.

\subsection{Classification}

Given a query image, we determine its location in the feature space and examine the nearby region. The closest training set images - or neighbours - are used to determine the category of the query image. If all neighbours represent the "arc" category, then the query image most likely also contains an arc. On the other hand, if there is no single dominant category among the neighbours, the classification of the query image is ambiguous.

As mentioned above, a single image feature does not usually provide good classification results and several features are needed. We used features dedicated for (1) auroral bright- ness, (2) north-south and (3) east-west aligned brightness distribution, and (4) a multi-scale texture measure. The resulting feature space is a hyperspace and the "meaning" of each basis is illustrated in Fig. 6. Once extracted, the features are normalised across the training data set. The total image-toimage distance is calculated as a weighted sum of individual feature distances. Mathematical details are given in the Appendix.

We utilised a variation of the K-nearest-neighbours (KNN) classifier for determining the contents of an auroral image. A KNN classifier outputs the class to which the majority of the neighbouring samples belongs to, and it has been shown that its error is never more than twice that of a Bayesian classifier, which is the optimal classifier in a statistical sense (Devroye et al., 1996). Since our stricter classifier provides an output class only if all neighbouring samples are in the same class, we can assume that its error should be smaller or equal to that of a standard KNN classifier, because all problematic cases are rejected and left without classification. 

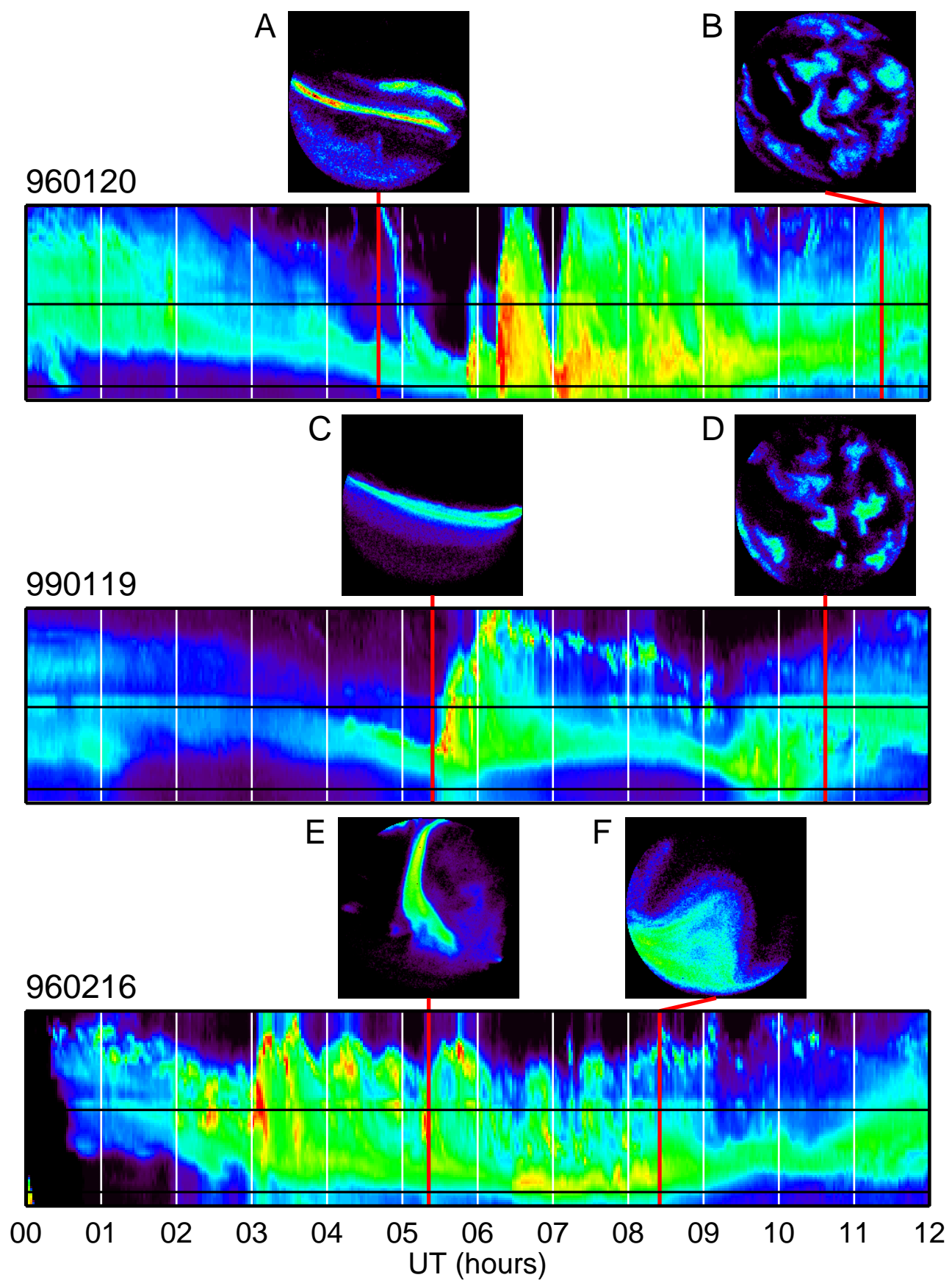

Fig. 3. Keograms from three nights constructed from $558 \mathrm{~nm}$ data collected by the CANOPUS Gillam and Rankin Inlet MSPs. The latitudinal extent of the ASI FOV is indicated by the two horizontal black lines. We include six $558 \mathrm{~nm}$ ASI images from Gillam, showing arcs (A) \& (C), patches (A) \& (D), a north-south aligned auroral form (E), and an Omega-band (F). 

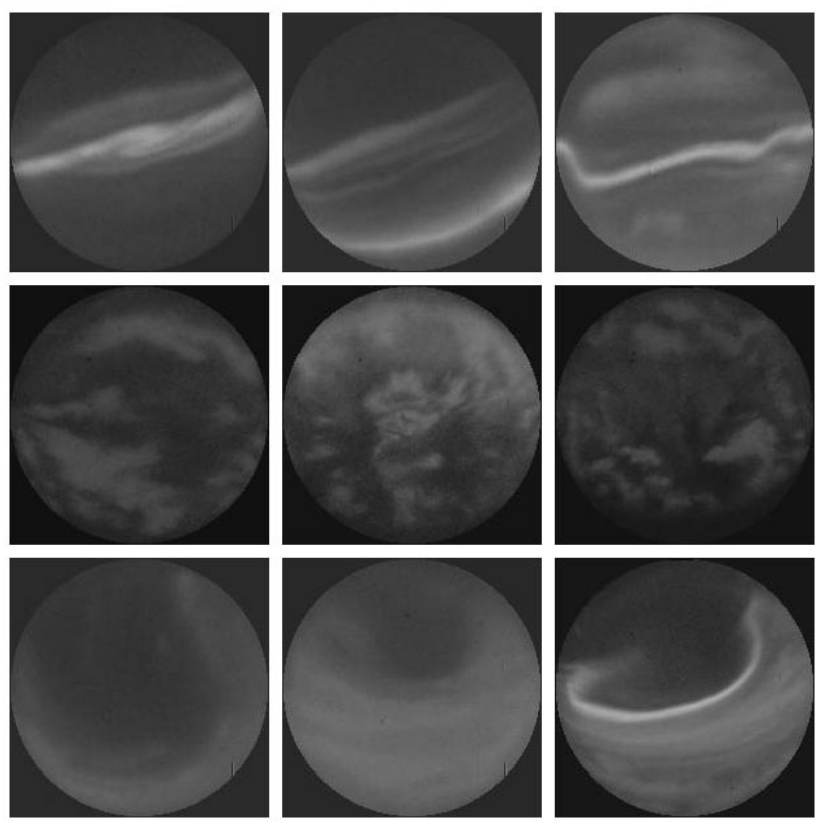

Fig. 4. Sample images from the three main auroral shape categories. Rows from top to bottom: auroral arcs, patchy auroras and Omegabands.

\subsection{Automated analysis of temporal distribution}

We used the previously described scheme to analyse several years worth of auroral images automatically in order to determine the relative temporal occurrence of arcs, patchy auroras and Omega-bands. For each image a unique ordering of the training set was determined based on the distances between the query and training images. Only if the five most similar images belonged to the same category, the query image was given the same classification. All other images were rejected (class "other"). This approach left us with only the strongest cases of occurrences for each shape category and neglected more ambiguous auroral appearances. Finally, the individual occurrence distributions were smoothed and normalised by the total number of all images with auroral activity for the same time instant.

Figure 7 shows the resulting distribution curves. The classification, which discarded ambiguous shapes, detected approximately 17000 auroral arcs, 9700 patchy auroras and 600 Omega-bands. Because we cannot determine the actual number of arcs, patchy auroras or Omega-bands in the whole image set, we studied only the relative occurrence within each category. Not surprisingly, auroral arcs occur mostly in the evening sector with a maximum at approximately 21 magnetic local time (MLT). Omega-bands and patchy auroras have clearly distinct maxima at 03:00 and 06:00 MLT, respectively.

\subsection{Error analysis}

The experimental error was determined by first re-classifying the training set. Here, one expects that each image should be
Table 1. The experimental error determined by comparing manual and automatic classification. First, all images in the training set (258 images) were classified automatically and the results were compared to their class label. Then, using the results from the automatic classification, 1700 randomly chosen images labelled as arcs (600 images), patchy auroras (600 images) and Omega-bands (500 images) were manually re-classified to determine the accuracy.

\begin{tabular}{ll} 
Image test set & Correct classification (\%) \\
\hline The training set with strict rules & $99 \pm 0.5$ \\
The training set set with standard KNN & $90 \pm 3$ \\
\hline Auroral arcs (593/600) & $99 \%$ \\
Patchy aurora (533/600) & $89 \%$ \\
Omega-bands (59/500) & $12 \%$
\end{tabular}

classified to the category it represents. The analysis indicated that for any practical purposes, the classifier can be considered errorless. For comparison, we also used the standard KNN classifier, in which all closest neighbours need not to be within one category. Now, even the problematic cases for example, neighbouring samples are two arcs and three patchy auroras - are classified instead of being rejected as shapes which are too ambiguous. This results in poorer performance with a $90 \pm 3 \%$ correct classification.

To evaluate the classifier performance in the full data set, we analysed 1700 randomly chosen images and compared their manual and automatic classifications. Arcs and patchy auroras were accurately classified, whereas Omega-bands are clearly more problematic. Table 1 summarises the error analysis.

\section{Discussion}

The image contents were analysed without using the temporal context - either in the form of (known) local time or by analysing the images as a sequence. The concept of "patchy auroras" is usually reserved for morning sector auroral shapes, and few auroral physicists would classify evening sector shapes as patchy auroras. However, individual images may resemble patchy auroras, especially in short-lived auroral activations. This is apparently also true for Omega-bands and leads to the slow rise in the respective distributions between 17:00 to 24:00 MLT.

Many auroral images share similar numeric features, which may provide conflicting information regarding their actual content category. This behaviour is verified by observing that only $12 \%$ of all ASI images (27 000 of 220000 ) could be classified into one single category with confidence. We studied images which could not be classified into a single category, and, indeed, many of them seemed to contain visual features from more than one auroral category or other shapes, such as breakup auroras. An auroral arc may have brighter sections, which naturally results in numerical similarity to both arcs and patchy auroras. 

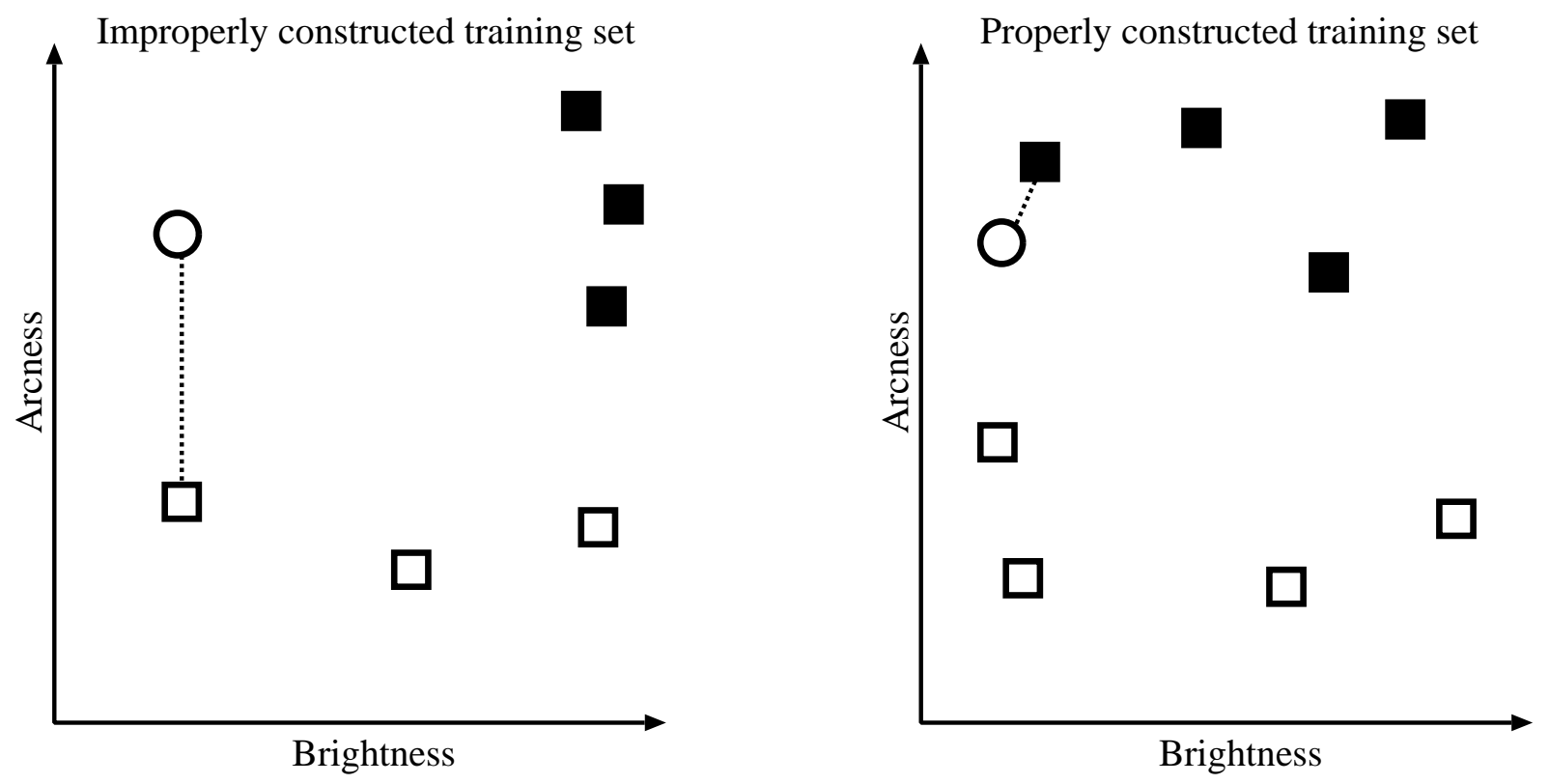

Fig. 5. Improperly constructed (left) and properly constructed (right) training set. The white circle denotes the query image while the squares denote the training set images with the black square being an image with an arc and the white square is one without an arc. The query image is connected to the nearest training set image by a dotted line. In the left panel, the query image is incorrectly classified as a non-arc. In the right panel, the training set has enough representative samples of arcs and the query image is correctly classified.

Feature 1

"Brightness"

"North-south"

"East-west"

"Texture"
Low contrast,

aurora not much brighter High contrast,

than background bright auroral shapes

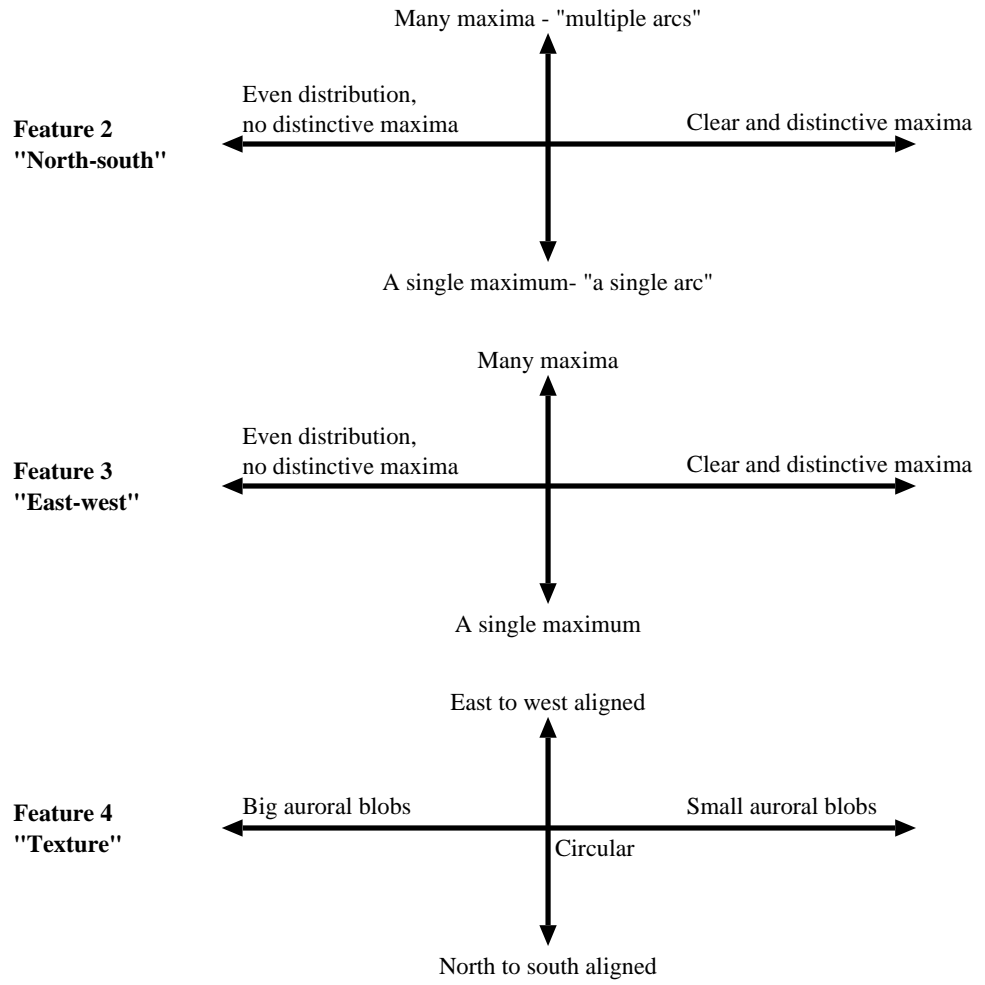

Fig. 6. Qualitative interpretation of the features. Feature 1 measures the overall contrast and distinctiveness of aurora in the image. Features 2 and 3 provide information about the distribution of the aurora - a clear maximum in north-south distribution, combined with an even distribution in east-west direction, usually represents an auroral arc. Similarly, many peaks presumably correspond to multiple arcs. Feature 4 is a proxy to "patchyness" or the size and shape of auroral blobs in the image. 


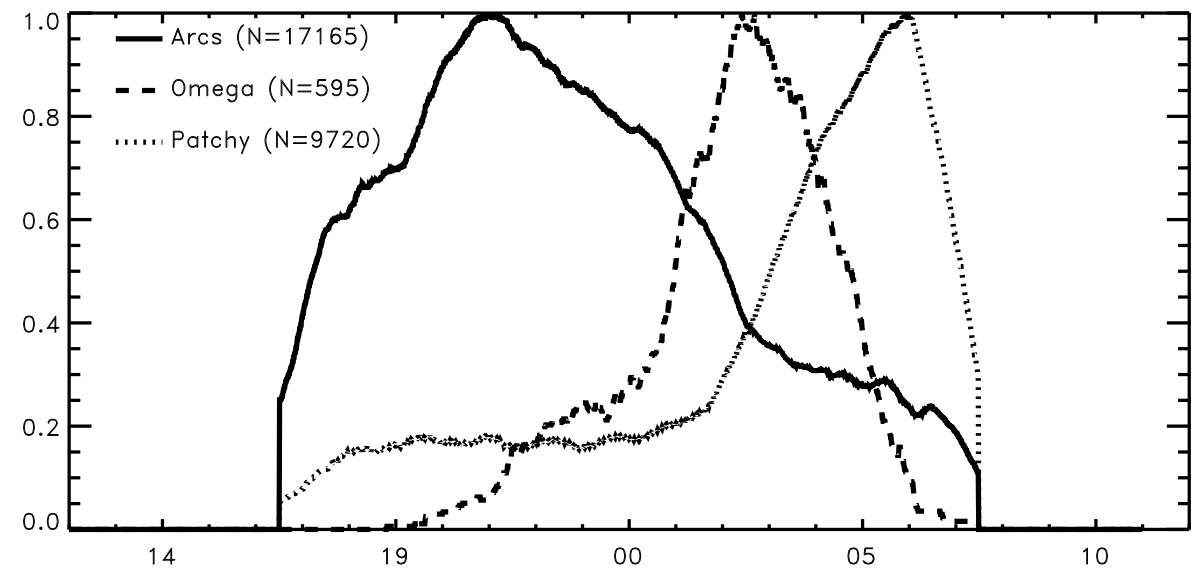

Fig. 7. Individual relative occurrences for auroral arcs, patchy auroras and Omega-bands vs. magnetic local time. The sharp cut-offs around 16:30 MLT and 07:30 MLT are due to local daylight. Note that the occurrences are individually normalised and do not represent relative occurrence frequency between arcs, patches and Omega-bands.
The accuracy of classification is very good when detecting arcs and patchy auroras. However, even though the classification for Omega-bands does not appear particularly successful, we noticed that the majority of the images reported as Omega-bands contained a boundary between diffuse aurora and background sky. If this boundary had looked like the Greek character $\Omega$, the classification would have been correct. One should also note that, while we used features specially designed for arcs and patchy auroras, we did not have an Omega-band feature that would utilise the shape of the boundary of background sky and aurora.

We did not have any training images for north-south aligned aurora in this study and we did not present any statistics for those shapes either. Intuitively, north-south structures and auroral arcs are apparently similar if either one is rotated $90^{\circ}$. The features for north-south and east-west aligned auroral distributions apparently provide enough information to recognise arcs, and we believe that adding north-south aligned auroral structures in the training set is definitely worth exploring in the future.

\section{Conclusions}

We have applied machine vision techniques in analysing the contents of 350000 auroral images automatically. First, one of the authors (EFD) provided a small training set consisting of sample images for different auroral shape categories. The automated process then examined a larger set of unlabelled images by searching for images similar to those in the training set. Content classification was accomplished by examining the category labels of the most similar images.

Studying the classification errors indicates that the automated analysis is consistent and accurate when classifying arcs and patchy auroras. However, there is a larger error in detecting Omega-bands. We believe that there are two major factor affecting the clearly worse classification with Omegabands: (1) small number of representative samples in the training set and (2) no dedicated feature to detect or verify the existence of Omega-band-like shapes in the aurora. Nevertheless, the location of the peak of relative Omega-band occurrence is in agreement with previous manual surveys.
Improving the training set would result in more accurate results by occupying more of the feature space in which image comparisons are performed. However, using some unsupervised clustering technique might prove to be even more fruitful: instead of relying on a naming tradition based on (subjective) auroral shape classification, automated analysis utilising millions of images could result in a more accurate spectrum of auroral forms.

In a recent study (Syrjäsuo et al., 2002), we searched for patchy auroras in the same CANOPUS data set using a different image-to-image distance measure. The search was performed in a content-based image retrieval fashion, in which the user provides a query image and the computer retrieves a fixed number of "similar" images (Rui et al., 1999; Smeulders et al., 2000). Essentially, the query image is analysed and the distance to all images in the data set is calculated. Then, the closest images are determined and these most similar images are then shown to the user. The performance of the more advanced classifier presented in this paper indicates that, in the near future, browsing auroral ASI data based on auroral shapes will be a realistic approach.

The required time for the actual classification operation for a single image is small enough to be implemented at the ASI stations in real time. Clearly, more studies are required, but one possible application is to capture images at increased temporal resolution and cull the resulting data on site. This would reduce the amount of data while maintaining superior temporal resolution during "interesting auroras". Another interesting research direction would be to study the time evolution of the aurora utilising motion in a well constructed feature space.

\section{Appendix A. Image features and similarity measure}

Let $\mathbf{I}(x, y)$ be an auroral image. Indices $i$ and $j$ denote the two images being compared. Each image is first rotated so that pixel rows are aligned to the (magnetic) east-west direction (direction of $x$ ). Correspondingly, pixels columns are in north-south direction $(y)$. The size of the image is $x_{\max } \times y_{\max }$ pixels. 


\section{A1 Brightness}

A heuristic intensity feature for auroral brightness is defined as

$\boldsymbol{f}_{1}=\left[\begin{array}{ll}\mu & b_{\max }\end{array}\right]^{T}$,

where $\mu$ is the average brightness, $b_{\max }$ the maximum brightness in the auroral image, and $T$ denotes transpose. The distance $S_{1}$ or similarity between two images is simply the Euclidean distance of the feature vectors

$S_{1}=\left\|\boldsymbol{f}_{1}^{(i)}-\boldsymbol{f}_{1}^{(j)}\right\|_{2}$.

\section{A2 Brightness distribution in (magnetic) N-S and E-W}

The feature representing the north-south distribution of auroral brightness essentially summarises the auroral brightness as a function of latitude within the FOV of the imager. However, we cannot simply average image rows because the FOV is circular. For each image row $y$, we determine the easternmost to the westernmost pixel columns within the FOV, denoted $C_{E}(y)$ and $C_{W}(y)$, as illustrated in Fig. A1. This is done to compensate for the circular FOV. Now, the average brightnesses in each row

$P(y)=\frac{1}{\left|C_{E}(y)-C_{W}(y)\right|} \sum_{x=C_{E}(y)}^{C_{W}(y)} \boldsymbol{I}(\boldsymbol{x}, \boldsymbol{y})$

are collected into a feature vector

$\boldsymbol{f}_{2}=\left[P(0) P(1) \ldots P\left(y_{\max }\right)\right]^{T}$.

In practise, the distribution is binned to 16 bins and normalised between $0 \ldots 1$. The distance is defined as

$S_{2}=\min _{k}\left\|\boldsymbol{f}_{2}^{(i)}-\operatorname{rot}\left(\boldsymbol{f}_{2}^{(j)}, k\right)\right\|$,

where $f_{2}$ denotes the 16-element feature vector and $\operatorname{rot}(\boldsymbol{f}, k)$ denotes a circular shift of vector elements by $k$ positions. The circular shift provides invariance in the northsouth direction: a bright arc in the north has a short distance to a bright arc in the south. The idea is illustrated in Fig. A2.

The distribution in east-west direction is defined similarly, as well as the corresponding distance measure $S_{3}$.

\section{A3 Texture}

The category of patchy aurora contains images in which the auroral classification should be based on the overall appearance instead of on a single identified auroral shape. Visually, patchy auroras resemble patterns of differently sized auroral "blobs". Depending on the size and orientation of these blobs, the patterns - called textures - vary. For identifying different textures, we used a multi-resolution Gabor-filter technique. Essentially, the elements of the texture feature vector $f_{4}$ can be associated with the response of 36 bandwidth filters that are tuned into different "auroral blob" scales and orientations. This is analogous to having "blob spectrum" and comparing the spectra of images. The distance $S_{5}$

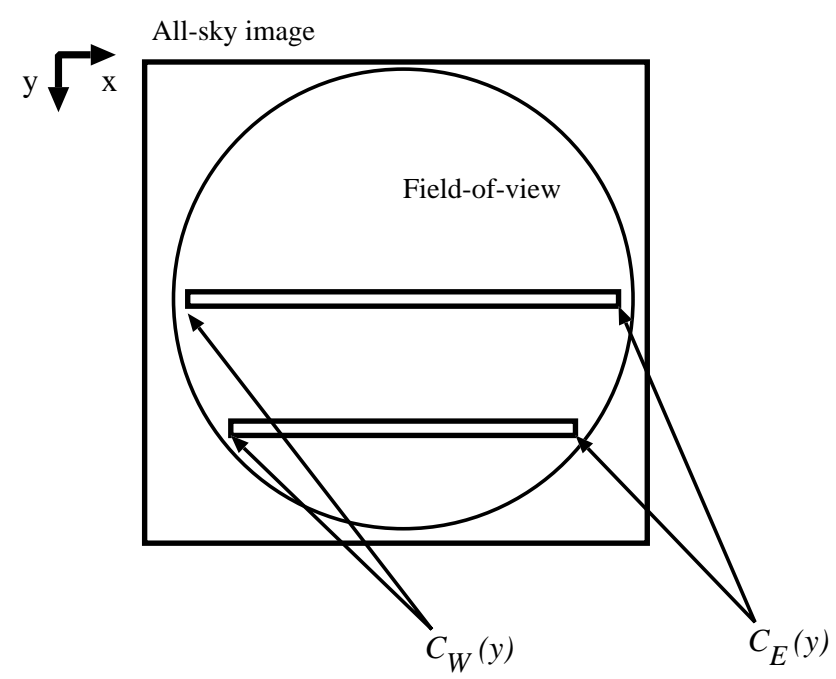

Fig. A1. Extracting brightness distribution: since the field-of-view produces a circular image, only a section of each row (east-west) contributes to the north-south aligned brightness profile. The easternmost and westernmost pixels for each row $y$ are $C_{E}(y)$ and $C_{W}(y)$.

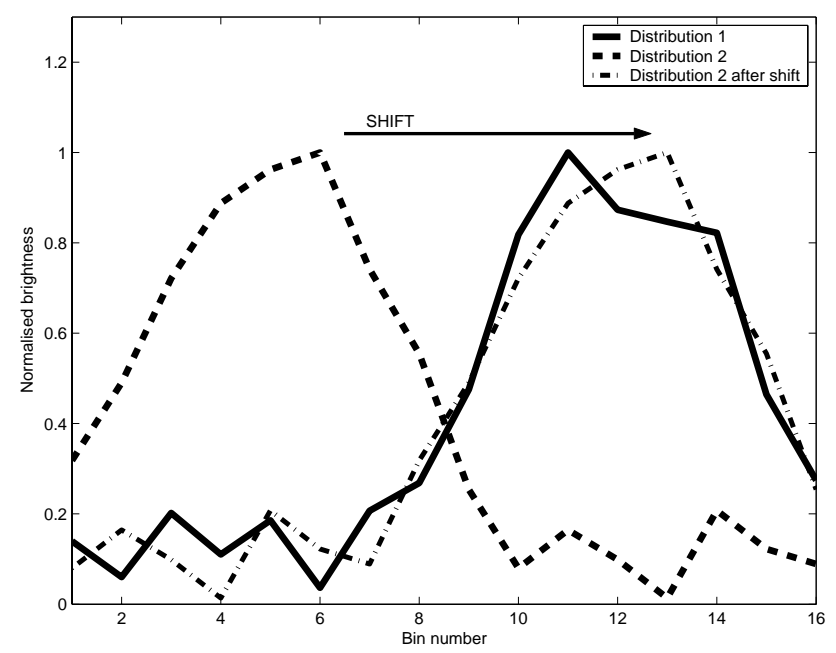

Fig. A2. The distance between two brightness distribution is the minimum distance obtained by circularly shifting one of the profiles. Distribution 1 in the plot is a result of having an arc south of zenith and Distribution 2 is another arc but north of zenith. By shifting Distribution 2, we obtain a better match - smaller distance - with Distribution 1, which is desirable because both brightness distributions represent a single arc. Each distribution is binned into 16 bins before distance calculation.

is small if the spectra - average blob size and orientation are similar. For details and the definition of the distance $S_{5}$, see Manjunath and Ma (1996) and references therein.

\section{A4 Feature weighting}

We used the iterative technique described in Rui et al. (1998) to determine the value of the weight vector $\boldsymbol{W}$ used in 
calculating the total image-to-image distance

$S=\boldsymbol{W}^{T}\left[\begin{array}{llll}S_{1} & S_{2} & S_{3} & S_{4}\end{array}\right]$

We used automated retrieving in the training image set to determine a weighting for features: because all images are already labelled, the similarity feedback for iterative weight adjustment is easily programmed. We simulated five retrievals with weight updating and averaged the weight vectors obtained by using each image in the labelled set as a query image.

Acknowledgements. CANOPUS is operated by the Canadian Space Agency and its Principal Investigator is L. L. Cogger. This work was financially supported by the Alberta Ingenuity Fund (Canada) and the Natural Sciences and Engineering Research Council (Canada).

Topical Editor M. Lester thanks K. Kaila and another referee for their help in evaluating this paper.

\section{References}

Akasofu, S.-I.: The auroral oval, the auroral substorm, and their relations with the internal structure of the magnetosphere, Planet. Space Sci., 14, 587-595, 1966.

Akasofu, S.-I. and Kimball, D.: The dynamics of the aurora 1. Instabilities of the aurora, J. Atmos. Terr. Phys., 26, 205-211, 1964.

Akasofu, S.-I., Kimball, D., Buchau, J., and Gowell, R.: Alignment of auroral arcs, J. Geophys. Res., 77, 4233-4236, 1972.

Devroye, L., Györfi, L., and Lugosi, G.: A probabilistic theory of pattern recognition, Springer, 1996.

Donovan, E. F., Trondsen, T. S., Cogger, L. L., and Jackel, B. J.: Auroral imaging in Canadian CANOPUS and NORSTAR programs, Proc. of the 30th Atmos. Studies by Optical Methods, in press, 2003.

Ejiri, M.: Trajectory traces of charged particles in the magnetosphere, J. Geophys. Res., 83, 4798, 1978.

Feldstein, Y.: Some problems concerning the morphology of auroras and magnetic disturbances at high latitudes, Geomag. Aeron., 3, 227, 1963.

Feldstein, Y. and Elphinstone, R.: Aurorae and the large-scale structure of the magnetosphere, J. Geomag. Geoelectr., 44, 11591174, 1992.

Gustafsson, G.: On the orientation of auroral arcs, Planet. Space Sci., 15, 277-294, 1967.

Henderson, M., Reeves, G., and Murphree, J.: Are north-south structures an ionopsheric manifestation of burstybulk flows?, Geophys. Res. Lett., 25, 3737-3740, 1998.

Kauristie, K., Syrjäsuo, M., Amm, O., Viljanen, A., Pulkkinen, T., and Opgenoorth, H.: Statistical study of evening sector arcs and electrojets, Advances in Space Research, 28, 1611-1616, 2001.

Knudsen, D., Donovan, E., Cogger, L., Jackel, B., and Shaw, W.: Width and structure of mesoscale optical auroral arcs, Geophys. Res. Lett., 28, 705-708, 2001.

Lyons, L., Fennell, J., and Vampola, A.: A general association between discrete auroras and ion precipitation from the tail, J. Geophys. Res., 93, 12 932-12 940, 1988.

Manjunath, B. and Ma, W.: Texture features for browsing and retrieval of image data, IEEE Trans. Pattern Analysis and Machine Intelligence, 18, 837-842, 1996.
Nevanlinna, H. and Pulkkinen, T.: Auroral observations in Finland: results from all-sky cameras, 1973-1977, J. Geophys. Res., 106, 8109-8118, 2001.

Opgenoorth, H., Oksman, J., Kaila, K., Nielsen, E., and Baumjohann, W.: Characteristics of eastward drifting Omega bands in the morning sector of the auroral oval, J. Geophys. Res., 99, 4115-4129, 1983.

Opgenoorth, H., Persson, M., Pulkkinen, T., and Pellinen, R.: Recovery phase of magnetospheric substorms and its association with morning sector aurora, J. Geophys. Res., 99, 4115-4129, 1994.

Rostoker, G., Samson, J. C., Creutzberg, F., Hughes, T. J., McDiarmid, D. R., McNamara, A. G. Vallance-Jones, A., Wallis, D. D., and Cogger, L. L.: CANOPUS - A ground-based instrument array for remote sensing of the high-latitude ionosphere during the ISTP/GGS Program, Space Sci. Rev., 71, 743-760, 1995.

Rui, Y., Huang, T., Ortega, M., and Mehrotra, S.: Relevance feedback: a power tool for interactive content-based image retrieval, IEEE Transactions on Circuits and Systems for Video Technology, 8, 644-655, 1998.

Rui, Y., Huang, T., and Chang, S.-F.: Image retrieval: current techniques, promising directions and open issues, J. of Visual Comm. and Image Representation, 39-62, 1999.

Samson, J., Lyons, L., Newell, P., Creutzberg, F., and Xu, B.: Proton aurora and substorm intensifications, Geophys. Res. Lett., 19, 2167-2170, 1992.

Sergeev, V. A., Sazhina, E. M., Tsyganenko, N. A., Lundblad, J.Å., and Søraas, F.: Pitch-angle scattering of energetic protons in the magnetotail current sheet as the dominant source of their isotropic precipitation into the nightside ionosphere, Planet. Space Sci., 31, 10, 1147-1155, 1983.

Smeulders, A., Worring, M., Santini, S., Gupta, A., and Jain, R.: Content-based image retrieval at the end of the early years, IEEE Trans. Pattern Analysis and Machine Intelligence, 22, 13491380, 2000.

Stringer, W. and Belon, A.: The morphology of the ISQY Auroral Oval 2. Auroral alignments in and near the auroral oval, J. Geophys. Res., 72, 4423-4429, 1967.

Syrjäsuo, M. and Donovan, E.: Analysis of auroral images: detection and tracking, Geophysica, 38, 3-14, 2002.

Syrjäsuo, M., Kauristie, K., and Pulkkinen, T.: Search engine for auroral forms, Adv. Space Res., 28, 1605-1610, 2001.

Syrjäsuo, M., Donovan, E., and Peura, M.: Using attribute trees to analyse auroral appearance over Canada, in IEEE Workshop on Applications of Machine Vision, 289-295, 2002.

Syrjäsuo, M. T., Pulkkinen, T. I., Janhunen, P., Viljanen, A., Pellinen, R. J., Kauristie, K., Opgenoorth, H. J., Wallman, S., Eglitis, P., Karlsson, P., Amm, O., Nielsen, E., and Thomas, C.: Observations of Substorm Electrodynamics by Using the MIRACLE Network, in: Proceedings of the Fourth International Conference on Substorms, edited by Kokobun, S. and Kamide, Y., 111-114, 1998.

Therrien, C. W.: Decision, estimation and classification, John Wiley \& Sons, 1989.

Yahnin, A., Sergeev, V., Gvozdevsky, B., and Vennerstrøm, S.: Magnetospheric source region of discrete auroras inferred from their relationship with isotropy boundaries of energetic particles, Ann. Geophys., 15, 943-958, 1997.

Zesta, E., Lyons, L., and Donovan, E.: The auroral signature of Earthward flow bursts observed in the magnetotail, Geophys. Res. Lett., 27, 3241-3244, 2000. 